\title{
Imunoexpressão do receptor do fator de crescimento epidérmico (EGFR) nos tecidos neoplásicos e adjacentes do carcinoma colorretal esporádico
}

Immunoexpression of the receptor epidermal growth factor receptor (EGFR) in tumor tissues and adjacents fron sporadic colorectal carcinoma

Bianca Doimo Sousa', Maurício Andrade Azevedo², Ana Maria Amaral Antonio Mader', Lourdes Conceição Martins', Jaques Waisberg ${ }^{5}$

\section{Resumo}

Introdução: 0 estudo da imunoexpressão tecidual do receptor do fator de crescimento epitelial (EGFR) pode contribuir para 0 entendimento de seu papel no prognóstico do carcinoma colorretal. Objetivo: Analisar a expressão imuno-histoquímica do EGFR no carcinoma colorretal e nos tecidos da transição tumor-mucosa e da mucosa adjacente à neoplasia. Métodos: Em 40 pacientes com carcinoma colorretal operados com intenção curativa, estudou-se a imunoexpressão do EGFR com anticorpo monoclonal anti-EGFR. Foram utilizados testes paramétricos e não paramétricos. Resultados: A imunoexpressão do EGFR nas amostras de tumor apresentou diferença significante em relação ao nível de imunoexpressão em espécimes de tecido da transição tumor/mucosa $(p=0,01)$ e no nível de imunoexpressão em tecidos da mucosa adjacente $(p=0,04)$. Conclusão: 0 EGFR apresentou maior imunoexpressão na mucosa do carcinoma colorretal em comparação à expressão no epitélio de transição e na mucosa adjacente não neoplásica.

Palavras-chave: Genes erbB-1; fator de crescimento epidérmico; marcadores biológicos; imunoistoquímica; carcinoma; neoplasias colorretais.

\begin{abstract}
Introduction: The study of tissue immunostaining epidermal growth factor receptor (EGFR) may contribute to the understanding of its role in the prognosis of colorectal carcinoma. Objective: To analyze the immunohistochemical expression of EGFR in colorectal carcinoma tissues and in tumor-mucosa and adjacent mucosal tissues to neoplasia Method: The expression of EGFR with an anti-EGFR monoclonal antibody was immunohistochemically assessed in colorectal tissue samples from 40 patients with colorectal sporadic adenocarcinoma operated on with curative intent. Parametric and non-parametric tests were used. Results: The immunohistochemical expression of EGFR in tumor samples showed a significant difference in the level of immunostaining in tissue specimens of transitional tumor/mucosa $(p=0.01)$ and the level of immunoreactivity in tissues of the adjacent mucosa $(p=0.04)$. Conclusion: The EGFR showed greatest expression in the mucosa of colorectal carcinoma than in the transitional epithelium and in adjacent non-neoplastic mucosa.
\end{abstract}

Keywords: Genes, erbB-1; epidermal growth factor; biological markers; immunohistochemistry; carcinoma; colorectal neoplasms.

Recebido em: 07/07/2011

Revisado em: 10/01/2012

Aprovado em: 20/01/2012

Trabalho realizado no Serviço de Gastroenterologia Cirúrgica do Hospital do Servidor Público Estadual (IAMSPE) - São Paulo (SP) e no Laboratório de Patologia Experimental da Faculdade de Medicina do ABC (FMABC) - Santo André (SP), Brasil.

${ }^{1}$ Curso de Medicina da Faculdade de Medicina do ABC (FMABC) - Santo André (SP), Brasil.

${ }^{2}$ Serviço de Gastroenterologia Cirúrgica do Conjunto Hospitalar do Mandaqui - São Paulo (SP), Brasil.

${ }^{3}$ Disciplina de Patologia da FMABC - Santo André (SP) Brasil.

4Programa de Pós-graduação em Saúde Coletiva da Universidade Católica de Santos (UNISANTOS) - Santos (SP), Brasil.

${ }^{5}$ Disciplina de Cirurgia do Aparelho Digestivo da FMABC - Santo André (SP), Brasil; Serviço de Gastroenterologia Cirúrgica do Hospital do Servidor Público Estadual (IAMSPE) - São Paulo (SP), Brasil.

Endereço para correspondência: Jaques Waisberg - Rua das Figueiras, 550, apto. 134 - Jardim Santo André - CEP: 09080-300 - Santo André (SP), Brasil -

E-mail: jaqueswaisberg@uol.com.br

Fonte de financiamento: B.D.S recebeu bolsa PIBIC - CNPq

Conflito de interesse: nada a declarar. 


\section{Introdução}

Nos países ocidentais, inclusive no Brasil, o carcinoma colorretal é o terceiro carcinoma mais frequente no mundo, cuja incidência está em elevação ${ }^{1,2}$. O estadiamento da neoplasia colorretal é, ainda, o fator prognóstico mais fidedigno, porém tal informação não é, habitualmente, disponível no pré-operatório33.

Para a tomada de decisão de se submeter ou não o paciente operado de carcinoma colorretal à quimioterapia pós-operatória é necessário selecionar os enfermos com prognóstico desfavorável, especialmente aqueles com lesões avançadas, e, nesse particular, a expressão dos marcadores tumorais no tecido neoplásico pode ser um método útil para esse propósito ${ }^{4-6}$.

Diversos marcadores teciduais são descritos na literatura, porém poucos apresentam relevância quanto à condução clínica do paciente $\mathrm{T}^{7,8}$. Idealmente, nas neoplasias colorretais, os marcadores teciduais deveriam alterar-se com o estádio do tumor, servir como exame prognóstico e auxiliar na definição da necessidade de realização de terapêutica complementar ${ }^{7,-12}$. Mesmo nos enfermos submetidos à ressecção da doença primária com intenção curativa, a recidiva neoplásica pós-operatória é uma frequente causa de óbito ${ }^{13-15}$.

A família de proteínas do fator de crescimento epidermal (EGF) inclui grupo de receptores e fatores de crescimento estruturalmente relacionados ${ }^{16-18}$. Muitas dessas proteínas, altamente expressadas em linhagens de células de câncer de cólon humano, ligam-se aos receptores EGF (EGFR) e desempenham um papel importante no crescimento do carcinoma colorretal ${ }^{16,19}$.

A expressão tecidual do EGFR pode ser determinada imuno-histoquímicamente pela ligação do EGF com a membrana do tumor ${ }^{19-24}$. No trato gastrintestinal, a expressão do EGFR é geralmente maior nos tumores do que nos tecidos normais ${ }^{25-27}$.

A hiperexpressão tecidual da EGFR é considerada indicadora de prognóstico clínico sombrio ${ }^{26,27}$, sugerindo progressão do carcinoma colorretal e potencial metastático ${ }^{26-29}$. No carcinoma colorretal, a hiperexpressão do EGFR foi relatada em 25-82\% dos pacientes e, nos pacientes com estádios avançados, revelou ser preditivo para o aparecimento de metástases à distância ${ }^{26-30}$. Entretanto, o impacto desse achado no prognóstico dos pacientes continua, ainda, controverso.

Estudos $^{31-34}$ falharam em mostrar relação entre o nível de expressão de EGFR e a eficiência clínica da terapia alvo. Essa discrepância alimentou explicações a respeito desse evento, incluindo a presença de heterogeneidade tumoral, sensibilidade baixa do método de detecção do EGFR e carência de metodologia padronizada nos estudos ${ }^{31,35,36}$. Assim, torna-se necessário definir os fatores que podem ser usados para identificar pacientes mais propensos a responder aos inibidores do EGFR para que tal tratamento possa ser escolhido ${ }^{37}$.

O objetivo deste estudo foi analisar a expressão do receptor do fator de crescimento epidérmico (EGFR) no tecido do carcinoma colorretal, em tecido da transição tumor-mucosa e em tecido da mucosa adjacente à neoplasia pela técnica imuno-histoquímica.

\section{Casuística e método}

Este estudo foi aprovado pelo Comitê de Ética em Pesquisa (CEP) do Instituto de Assistência Médica ao Servidor Público Estadual (IAMSPE), parecer $\mathrm{n}^{\circ} 062 / 08$.

No período de outubro de 2005 a março de 2007 foram estudados 40 pacientes com carcinoma colorretal operados com intenção curativa no Serviço de Gastroenterologia Cirúrgica do Hospital do Servidor Público Estadual de São Paulo (IAMSPE). Desses pacientes, $20(50 \%)$ eram homens e 20 (50\%) mulheres. A média de idade foi de $68,7 \pm 11,6$ anos (44 a 90 anos).

Nesse estudo foram considerados critérios de inclusão a presença de carcinoma colorretal confirmada pelo estudo histopatológico e lesão extirpada com intenção curativa. Foram considerados critérios de exclusão pacientes com menos de 18 anos de idade, portadores de doença inflamatória intestinal, portadores de neoplasia em outros órgãos ou lesão extirpada de modo paliativo.

O estadiamento pré-operatório foi realizado pelo exame clínico e proctológico completos, determinação sérica do antígeno carcinoembrionário (CEA), colonoscopia com biópsia e estudo histopatológico da lesão e tomografia computadorizada de tórax e abdome. Foi utilizado o estadiamento do carcinoma colorretal proposto pelo American Joint Cancer Committee (AJCC) de 2010.

Para a realização do estudo histopatológico foram obtidos três espécimes: uma da área central do tumor, evitando-se locais ulcerados ou com necrose; outra da região de transição entre a neoplasia e a área macroscopicamente não tumoral com confirmação microscópica dessa área transicional e uma terceira amostra obtida de mucosa adjacente localizada a $10 \mathrm{~cm}$ da lesão. Todos os espécimes cirúrgicos previamente fixados em solução de formol a 10\% foram incluídos em blocos de parafina. Três cortes de $4 \mu \mathrm{m}$ foram obtidos de cada bloco para a obtenção de áreas com neoplasia, área de transição neoplasia/mucosa macroscopicamente não tumoral e da área macroscopicamente sem neoplasia. Todos os espécimes foram corados pela técnica de hematoxilina-eosina (HE) para estudo microscópico e verificação do comprometimento neoplásico dos linfonodos ressecados e das margens cirúrgicas.

Os blocos de parafina foram seccionados com $3 \mu \mathrm{m}$ de espessura e as lâminas submetidas à técnica imuno-histoquímica $\mathrm{ABC}$ (complexo avidina-biotina-peroxidase) com o anticorpo primário anti-EGFR na diluição 1:30 (mouse monoclonal anti-human epidermal growth factor receptor - EGFR, lote 3360, clone H11, Dako Cytomation, EUA).

Foi considerada reação positiva para o anticorpo EGFR o aparecimento da cor marrom na área citoplasmática ou membranosa da célula. Os controles positivos foram cortes do centro germinativo de tonsila normal. Nas lâminas usadas como controle negativo subtraiu-se o anticorpo primário da reação.

A leitura das lâminas foi feita por patologista experiente, utilizando-se microscópio binocular da marca Nikon, com objetivas planacromáticas. Inicialmente, foram selecionadas as áreas mais positivas 
(hot spots) sob o aumento de $100 \mathrm{X}$ e, em seguida, passou-se para o aumento de $400 \mathrm{X}$, com avaliação de 10 campos consecutivos. A imunoexpressão do EGFR, representada por colorido acastanhado inequívoco tanto no citoplasma (Figura 1) como na membrana citoplasmática (Figura 2) das células neoplásicas, foi avaliada de modo semiquantitativo, segundo os critérios propostos por Kountourakis et al. ${ }^{38}$. No citoplasma, a imunoexpressão foi classificada como zero: sem coloração ou $<10 \%$ das células neoplásicas com fraca intensidade; + : $>10 \%$ das células com fraca intensidade; $++:>10 \%$ das células com média intensidade; $+++:>10 \%$ das células de forte intensidade. Na membrana citoplasmática, a imunoexpressão foi classificada como zero: sem coloração; +: $<10 \%$ de células neoplásicas de qualquer intensidade ou $<30 \%$ de células de fraca intensidade; ++: 10-30\% de células com média a forte intensidade ou $30-50 \%$ de células neoplásicas com fraca a média intensidade; $+++:>30 \%$ de células com forte intensidade ou $>50 \%$ de células neoplásicas com qualquer intensidade de coloração.

A avaliação da imunoexpressão do EGFR foi realizada no tumor, no tecido da transição tumor-mucosa não neoplásica e na mucosa adjacente a $10 \mathrm{~cm}$ cranialmente à lesão neoplásica.

Para a análise dos resultados, os pacientes foram divididos em dois grupos: grupo zero, onde não houve imunoexpressão do EGFR e grupo um, com imunoexpressão do EGFR, independente da sua intensidade.

Os dados referentes às variáveis quantitativas foram apresentados em médias. As variáveis categóricas foram analisadas pelo teste de Mann-Witney e a correlação entre elas foi realizada pelo teste de Spearman. A análise de variância nas amostras de três ou mais grupos foi obtida pelo teste de Kruskal-Wallis. As variáveis dicotômicas foram analisadas pelo teste exato de Fisher.

O programa estatístico utilizado foi o Prism 4.0 (GraphPad Software Inc., EUA) e o nível de significância adotado foi inferior a 0,05 ou $5 \%$.

\section{Resultados}

As lesões estavam localizadas no cólon em onze (37,93\%) pacientes e no reto em $18(62,07 \%)$ enfermos.

A operação mais executada foi a retossigmoidectomia em 24 $(60 \%)$ pacientes, seguida da colectomia total em 5 (12,5\%), colectomia esquerda em 5 (12,5\%), colectomia direita em 5 (12,5\%) e amputação abdominoperineal do reto em 1 (2,5\%).

Verificou-se que a média do tamanho da neoplasia colorretal foi de $4,25 \pm 2,1 \mathrm{~cm}(1,0-9,0 \mathrm{~cm})$. Vinte e um (52,5\%) pacientes tiveram lesões com diâmetro $\leq 5,0 \mathrm{~cm}$, enquanto que outros $19(47,5 \%)$ apresentaram neoplasia com diâmetro $>5,0 \mathrm{~cm}$. Doze (30\%) pacientes apresentavam invasão vascular sanguínea pela neoplasia no exame anatomopatológico da peça extirpada, enquanto em 28 (70\%) enfermos não foi constatada invasão vascular. Quatorze (35\%) pacientes apresentavam invasão vascular linfática constatada no exame anatomopatológico da peça

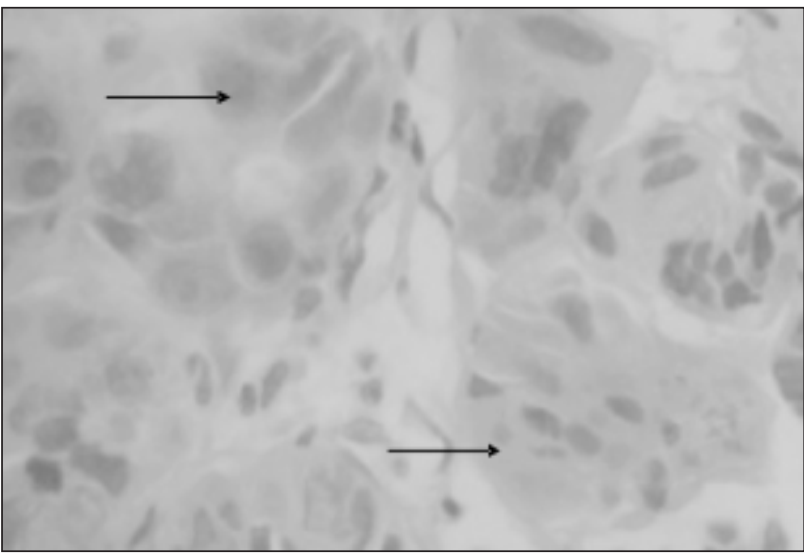

Figura 1: Imunoexpressão de padrão citoplasmático de média de intensidade do EGFR nas células neoplásicas (Imuno-histoquímica; $400 \mathrm{X})$.

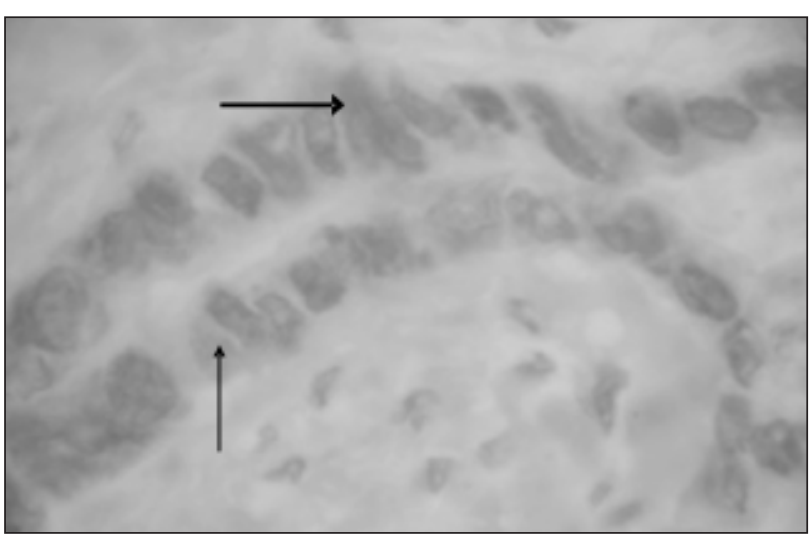

Figura 2: Imunoexpressão de padrão membranoso e forte intensidade do EGFR nas células neoplásicas (Imuno-histoquímica; $400 \mathrm{X}$ ).

extirpada, enquanto em 26 (65\%) enfermos a neoplasia não apresentava invasão vascular linfática. Sete $(17,5 \%)$ pacientes apresentavam invasão perineural constatada no exame anatomopatológico da peça extirpada, enquanto 33 (82,5\%) enfermos não exibiam neoplasia com invasão perineural. Houve acometimento pelo carcinoma colorretal dos linfonodos extirpados em 19 (47,5\%) pacientes. Em outros 21 (52,5\%) enfermos, os linfonodos da peça operatória estavam livres de acometimento neoplásico. No tocante ao grau de diferenciação celular do carcinoma colorretal, 31 (77,5\%) pacientes exibiam adenocarcinoma moderadamente diferenciado, 2 (5\%) adenocarcinoma pouco diferenciado e 7 (17,5\%) adenocarcinoma bem diferenciado. A lesão penetrava a serosa (peritônio visceral) sem invasão das estruturas adjacentes (baço, estômago, fígado, diafragma, pâncreas, parede abdominal, adrenal, rim, intestino delgado e retroperitônio) (T3) em 28 (70\%) enfermos; a lesão invadia a muscular própria ou subserosa (T2) em 10 (25\%) pacientes, e estruturas adjacentes (T4) em 2 (5\%) dos pacientes. Nenhum dos enfermos apresentou somente invasão da lâmina própria ou submucosa (T1). Quatro (10\%) pacientes apresentavam metástases hepáticas constatadas no momento da operação, enquanto 36 (90\%) enfermos não exibiam metástases hepáticas. 
Tabela 1: Presença ou ausência de imunoexpressão do EGFR nas amostras de tecido tumoral, tecido da transição tumor/mucosa e tecido da mucosa adjacente dos pacientes com carcinoma colorretal

\begin{tabular}{lccc}
\hline Tipo de tecido & $\mathrm{n}$ & $\begin{array}{c}\text { Imunoexpressão } \\
\text { do EGFR presente } \\
\mathrm{n}(\%)\end{array}$ & $\begin{array}{c}\text { Imunoexpressão } \\
\text { do EGFR ausente } \\
\mathrm{n}(\%)\end{array}$ \\
\hline Tecido tumoral & 40 & $18(45 \%)$ & $22(55 \%)$ \\
Transição tumor/mucosa & 40 & $14(35 \%)$ & $26(65 \%)$ \\
Mucosa adjacente & 40 & $11(27,5 \%)$ & $29(72,5 \%)$ \\
\hline
\end{tabular}

n: Número de pacientes

Tabela 2: Comparação entre a presença de imunoexpressão do EGFR no tecido tumoral, tecido da transição tumor/mucosa e no tecido da mucosa adjacente dos pacientes com carcinoma colorretal

\begin{tabular}{lcc}
\hline Tipo de tecido & Imunoexpressão do EGFR & $\mathrm{p}$ \\
\hline Tecido tumoral & $\mathrm{n}=18(45 \%)$ & $0,01^{*}$ \\
Transição tumor-mucosa & $\mathrm{n}=14(35 \%)$ & $0,04^{*}$ \\
Mucosa adjacente & $\mathrm{n}=11(27,5 \%)$ & $0,01^{*}$ \\
\hline
\end{tabular}

$\mathrm{n}$ : Número de pacientes

*Teste de Mann-Witney

Verificou-se que nas amostras do tecido tumoral a imunoexpressão do EGFR estava ausente em 22 (55\%) pacientes e presente em 18 (45\%). Em relação às amostras do tecido da transição tumor-mucosa não neoplásica, houve presença de imunoexpressão do EGFR em $14(35 \%)$, ao passo que 26 (65\%) das amostras teciduais não exibiram imunoexpressão do EGFR. No tocante à imunoexpressão do EGFR no tecido da mucosa adjacente, constatou-se que 11 (27,5\%) pacientes tiveram amostras teciduais com imunoexpressão e $29(72,5 \%)$ não apresentaram imunoexpresão (Tabela 1).

A imunoexpressão do EGFR no tecido neoplásico foi significantemente maior $(\mathrm{p}=0,01)$ que no tecido da transição tumor-mucosa. Da mesma forma, a imunoexpressão do EGFR foi significantemente maior $(\mathrm{p}=0,04)$ no tecido neoplásico quando comparado à mucosa adjacente. Observou-se também que a imunoexpressão do EGFR no tecido da transição tumor-mucosa foi significantemente maior $(p=0,01)$ em relação à imunoexpressão do EGFR na mucosa adjacente (Tabela 2).

\section{Discussão}

Por um lado, o presente estudo foi realizado de forma retrospectiva, o que permitiu a ocorrência de eventuais vieses próprios desse tipo de análise, por outro, a padronização da coleta dos dados e do estudo anatomopatológico e a análise estatística criteriosa permitiram a redução desses eventuais vieses.

O marcador EGFR foi escolhido para identificar dados relevantes da carcinogênese da neoplasia colorretal. Trata-se de marcador cuja expressão aumentada no tecido relaciona-se com prognóstico mais sombrio e com a presença de metástases hepáticas. No entanto, a literatura é controversa quanto ao significado do aumento da expressão desse marcador $28,30,31,33,35,37$.

A expressão do marcador EGFR no presente estudo foi significantemente maior no tecido neoplásico que nos espécimes de tecido da transição carcinoma/mucosa e nos tecidos da mucosa adjacente. Sendo o EGFR receptor do tipo tirosina-quinase, ele tem participação na ativação nas cascatas relacionadas à diferenciação e à multiplicação celular ${ }^{39}$. Goldstein e Arrmin ${ }^{26}$ identificaram a hiperexpressão do EGFR nas camadas mais profundas do tumor e sua associação com o aparecimento de metástases hepáticas e linfonodais. Esses autores identificaram expressão maior do EGFR no tecido neoplásico em relação à mucosa não neoplásica adjacente ao tumor. Observou-se, no presente estudo, que as diferentes medidas da imunoexpressão (índice de positividade e intensidade de expressão) apresentaram diferenças significantes nas regiões do centro do tumor, transição tumor-mucosa e mucosa adjacente. Admitindo-se que a imunoexpressão apresenta relação direta com a quantidade EGFR presente em determinada amostra de tecido, esse achado sugere que o conteúdo do EGFR é maior no tecido neoplásico quando comparado com o tecido da transição tumor/mucosa não neoplásica e com o tecido da mucosa adjacente do carcinoma colorretal.

$\mathrm{Na}$ presente série foram realizadas análises em tecido neoplásico em espécimes de tecido da transição carcinoma/mucosa e em espécimes de tecido de mucosa não neoplásica adjacente ao tumor. Embora a mucosa não neoplásica retirada cuidadosamente a $10 \mathrm{~cm}$ do local do tumor fosse considerada sem alterações, é possível que ela contivesse afinidade diferente ao marcador da mucosa de pacientes sem carcinoma colorretal. No entanto, outro estudo ${ }^{23}$ considerou como mucosa "normal" aquela adjacente ao tumor e sem tecido tumoral identificável microscopicamente.

O estudo por meio da imuno-histoquímica foi realizado em função da aplicabilidade do método e do seu baixo custo, além de esse método ser bem estabelecido na literatura específica ${ }^{16,38}$.

A análise imuno-histoquímica do tecido do carcinoma colorretal foi realizada pelo método semiquantitativo, sendo a expressão dos marcadores no tecido tumoral e na mucosa normal avaliada pelo número de células coradas e pela intensidade da coloração.

A interpretação da expressão dos marcadores foi realizada quantitativamente conforme a técnica de Kountourakis et al. ${ }^{38}$. Inicialmente, foram selecionadas as áreas mais positivas (hot spots) sob o aumento de $100 \mathrm{X}$ e, em seguida, passou-se para o aumento de $400 \mathrm{X}$, com avaliação de dez campos consecutivos. A imunoexpressão do EGFR, representada por colorido acastanhado inequívoco tanto no citoplasma como na membrana citoplasmática das células neoplásicas, foi avaliada, de modo semiquantitativo, no citoplasma. Como esses resultados foram obtidos por método validado, foram considerados válidos e utilizados no presente estudo.

Nesse estudo não houve diferença significante na expressão do EGFR de acordo com a localização retal ou cólica do carcinoma. Esse 
mesmo achado foi observado por Spano et al. ${ }^{27}$, Kountourakis et al. ${ }^{38} \mathrm{e}$ Baiocchi et al..$^{40}$. Esse resultado pode indicar que a imunoexpressão do EGFR independe das características morfológicas das diferentes regiões do intestino grosso, quando se compara o colo com o reto.

Nas condições deste estudo, os resultados obtidos permiram concluir que a imunoexpressão do EGFR foi mais intensa na mucosa do carcinoma colorretal que no epitélio de transição tumor-mucosa e na mucosa adjacente não neoplásica. Estudos adicionais são necessários para avaliar a eventual relação entre a expressão imuno-histoquímica do EGFR e o prognóstico do carcinoma colorretal, e se a expressão imuno-histoquímica do EGFR pode ser útil como marcador preditivo para que o paciente obtenha resultados benéficos com a quimioterapia.

\section{Referências}

1. Jemal A, Siegel R, Ward E, Murray T, Xu J, Smigal C, et al. Cancer Statistics; 2006. CA Cancer J Clin. 2006;56(2):106-30

2. American Cancer Society [Internet]. Global Cancer Facts \& Figures 2007. [cited 2011 Jul 08]. Available from: http://www.cancer.org/acs/groups/ content/@nho/documents/document/globalfactsandfigures2007rev2p.pdf

3. Louvet C, Gramont A, Tournigand C, Artru P, Maindrault-Goebel F, Krulik M. Correlation between progression free survival and response rate in patients with metastatic colorectal carcinoma. Cancer. 2001;91(11):2033-8.

4. Midgley RS, Kerr DJ. Adjuvant treatment of colorectal cancer. Cancer Treat Rev. 1997;23(3):135-52

5. Sobrero A, Guglielmi A. Current controversies in the adjuvant therapy of colon cancer. Ann Oncol. 2004;15(Suppl 4):iv39-41.

6. Zaniboni A, Labianca R. Grupo italiano per lo studio e la cura dei tumori del digerente. Adjuvant therapy for stage II colon cancer: an elephant in the living room? Ann Oncol. 2004;15(9):1310-8.

7. Bhatavdekar JM, Patel DD, Chikhlikar PR, Shah NG, Vora HH, Glosh N, et al. Molecular markers are predictors of recurrence and survival in patients with Dukes B and Dukes C colorectal adenocarcinoma. Dis Colon Rectum. 2001:44(4):523-33.

8. Waisberg J, Landman G, Cha ASH, Henriques AC, Gaspar HA, Speranzini MB. Padrão da distribuição tecidual do CEA no carcinoma colo-retal: relação com o nível sérico do CEA e classificação de Dukes. Rev Bras Colo-Proctol. 2002;22(1):20-6.

9. Levy M, Visokai V, Lipska L, Topolcan 0. Tumor markers in staging and prognosis of colorectal carcinoma. Neoplasma. 2008;55(2):138-42.

10. Lyall MS, Dundas SR, Curran S, Murray GI. Profiling markers of prognosis in colorectal cancer. Clin Cancer Res. 2006;12(4):1184-91.

11. Galizia G, Lieto E, Ferraracio F, Orditura M, Vita F, Castellano P, et al. Determination of molecular marker expression can predict clinical outcome in colon carcinomas. Clin Cancer Res. 2004;10(10):3490-9

12. Bukeirat FA, Ostrinsky Y, McFadden D. Use of GI tumor markers in clinical practice: a practical interpretation. W V Med J. 2008;104(4):17-21.

13. Duffy MJ, Dalen A, Haglund C, Hansson L, Holinski-Feder E, Klapdor R, et al. Tumour markers in colorectal cancer: European Group on Tumour Markers (EGTM) guidelines for clinical use. Eur J Cancer. 2007:43(9):1348-60.

14. Daniels IR, Fisher SE, Heald RJ, Moran BJ. Accurate staging, selective preoperative therapy and optimal surgery improves outcome in rectal cancer: a review of the recent evidence. Colorectal Dis. 2007;9(4):290-301.
15. Stein U, Schlag PM. Clinical, biological, and molecular aspects of metastasis in colorectal cancer. Recent Results Cancer Res. 2007:176:61-80

16. Brand TM, lida $M$, Li C, Wheeler DL. The nuclear epidermal growth factor receptor signaling network and its role in cancer. Discov Med. 2011;12(66):419-32.

17. Grossmann AH, Samowitz WS. Epidermal growth factor receptor pathway mutations and colorectal cancer therapy. Arch Pathol Lab Med. 2011;135(10):1278-82.

18. Enrique AA, Gema PC, Jeronimo JC, Auxiliadora GE. Role of anti-EGFR target therapy in colorectal carcinoma. Front Biosci. 2012;4:12-22.

19. Yano S, Kondo K, Yamaguchi M, Richmond G, Hutchison M, Wakeling A et al. Distribution and fuction of EGFR in human tissue and the effect of EGRF tyrosine kinase inibition. Anticancer Res. 2003;23(5A):3639-50.

20. Grandis JR, Sok JC. Signaling through the epidermal growth factor receptor during the development of malignancy. Pharmacol Ther 2004;102(I):37-46.

21. Mendelsohn J, Baselga J. Status of epidermal growth factor receptor antagonists in the biology and treatment of cancer. J Clin Oncol. 2003:21(14):2787-99.

22. Roberts RB, Min L, Washington MK, Olsen SJ, Settle SH, Coffey RJ, et al Importance of epidermal growth factor receptor signaling in establishment of adenomas and maintenance of carcinomas during intestinal tumorigenesis. Proc Natl Acad Sci U S A. 2002;99(3):1521-6.

23. Alferez D, Wilkinson RW, Watkins J, Poulsom R, Mandir N, Wedge SP, et al. Dual inhibition of VEGFR and EGFR signaling reduces the incidence and size of intestinal adenomas in Apc (Min/+) mice. Mol Cancer Ther 2008;7(3):590-8.

24. Sergina NV, Moasser MM. The HER family and cancer: emerging molecular mechanisms and therapeutic targets. Trends Mol Med. 2007;13(12):527-34.

25. Ciardiello F, Tortora G. A novel approach in the treatment of cancer targeting the epidermal growth factor receptor. Clin Cancer Res. 2001;7(10):2958-70

26. Goldstein NS, Armin M, Epidermal growth factor receptor immunohistochemical reactivity in patients with American Joint Committee on Cancer Stage IV colon adenocarcinoma: implications for a standardized scoring system. Cancer. 2001;92(5):1331-46.

27. Spano JP. Lagorce C, Atlan D, Milano G, Domont J, Benamouzing R, et al. Impact of EGFR expression on colorectal cancer patient prognosis and survival. Ann Oncol. 2005;16(1):102-8. 
28. Italiano A, Saint-Paul MC, Caroli-Bosc FX, François E, Bourgeon A, Benchimol $\mathrm{D}$, et al. Epidermal growth factor (EGFR) status in primary colorectal tumors correlates with EGFR expression in related metastatic sites: biological and clinical implications. Ann Oncol. 2005;16(9):1503-07.

29. Khalifa MA, Rowsell CH, Gladdy RA, Ko YJ, Hanna S, Smith A, et al. Expression of epidermal growth factor receptor in primary colorectal adenocarcinoma predicts expression in recurrent disease. Am J Clin Pathol. 2006;125(2):229-33.

30. Bralet MP, Paule B, Falissard B, Adam R, Guettier C. Immunohistochemical variability of epidermal growth factor receptor (EGFR) in liver metastases from colonic carcinomas. Histopathology. 2007;50(2):210-6.

31. Scartozzi M, Bearzi I, Bearzi R, Mandolesi A, Fabris G, Cascinu S. Epidermal growth factor receptor (EGFR) status in primary colorectal tumors does not correlate with EGFR expression in related metastatic sites: implication for treatment with EGFR-targeted monoclonal antibodies. J Clin Oncol. 2004;22(23):4772-8.

32. Galizia G, Lieto E, Ferraraccio F, Vita F, Castellano P, Orditura M, et al. Prognostic significance of epidermal growth factor receptor expression in colon cancer patients undergoing curative surgery. Ann Surg Oncol. 2006;13(6):823-35.

33. Cunningham D, Humblet $Y$, Siena S, Khayat D, Bleiberg H, Santoro A, et al. Cetuximab monotherapy and cetuximab plus irinotecam in irinotecanrefractory metastatic colorectal câncer. N Engl J Med. 2004;351(4):337-45.
34. Overman MJ, Hoff PM. EGFR-target therapies in colorectal cancer. Dis Colon Rectum. 2007:50(8):1259-60.

35. Doger FK, Meteoglu I, Tuncyurek P, Okyay P, Cevikel H. Does the EGFR and VEGF expression predict the prognosis in colon cancer? Eur Surg Res. 2006;38(6):540-4

36. Spindler KL, Lindebjerg J, Nielsen JN, Olsen DA, Bisgard C, Brandslund I, et al. Epidermal growth factor receptor analyses in colorectal cancer: a comparison of methods. Int J Oncol. 2006;29(5):1159-65.

37. Ciardiello F, Tortora G. EGFR antagonists in cancer treatment. N Engl J Med. 2008;358(11):1160-74.

38. Kountourakis P, Pavlakis K, Psyrri A, Rontogianni D, Xiros N, Patsouris E, et al. Clinicopathologic significance of EGFR and Her-2/neu in colorectal adenocarcinomas. Cancer J. 2006;12(3):229-36.

39. Karameris A, Kanavaros P, Aninos D, Gorgoulis V, Mikou G, Rokas T, et al. Expression of epidermal growth factor (EGF) and epidermal growth factor receptor (EGFR) in gastric and colorectal carcinomas. An immunohistological study of 63 cases. Pathol Res Pract. 1993;189(2):133-7.

40. Baiocchi G, Lopes A, Couldry RA, Rossi BM, Soares FA, Aguiar S, et al. ErbB family immunohistochemical expression in colorectal cancer patients with higher risk of recurrence after radical surgery. Int J Colorectal Dis. 2009;24(9):1059-68. 